\title{
Electrocatalytic Properties of BDD Anodes: Its Loosely Adsorbed Hydroxyl Radicals
}

\author{
Nicolaos Vatistas \\ DICCISM, University of Pisa, Via Diotisalvi, 2, 6126 Pisa, Italy \\ Correspondence should be addressed to Nicolaos Vatistas, n.vatistas@diccism.unipi.it \\ Received 1 May 2011; Accepted 6 July 2011 \\ Academic Editor: Giancarlo R. Salazar-Banda \\ Copyright () 2012 Nicolaos Vatistas. This is an open access article distributed under the Creative Commons Attribution License, \\ which permits unrestricted use, distribution, and reproduction in any medium, provided the original work is properly cited.
}

The high oxidative action of boron doped diamond (BDD) anodes on the biorefractory organic compounds has been attributed to the low adsorption of the generated hydroxyl radicals on the BDD surface in respect to other anodic materials. In a previous paper, the effect of low adsorption of BBD has been studied by proposing a continuum approach to represent the adsorption layer. The oxidative action of the hydroxyl radicals is attributed to the values of their diffusivity into the adsorption and adjacent reactive layer as well as to the value of kinetic constant in both layers. In this paper, more details on both layers are reported in order to justify the proposed continuum approach as well as the assumptions concerning diffusivity and kinetic constant in both adsorption and reactive layers, where the oxidative action of hydroxyl radicals occurs.

\section{Introduction}

The BDD anodes have particular electrocatalytic properties in respect to others, that are, oxygen is generated on its surface, when the anodic overpotential achieves higher values, biorefractory organic species are efficiently eliminated, and peroxide cation are efficiently produced. These specific electrocatalytic properties are attributed to a specific property of the generated hydroxyl radicals $\mathrm{HO}^{*}$ on BDD electrodes in respect to other electrodes [1] that is, these radicals are less adsorbed on the surface of $\mathrm{BDD}$ anodes than on the surface of other anodes. In fact, the experimentally detected increase of oxidative action of the anodes $\mathrm{RuO}_{2}-\mathrm{TiO}_{2}, \mathrm{IrO}_{2}-\mathrm{Ta}_{2} \mathrm{O}_{5}, \mathrm{Pt}, \mathrm{PbO}_{2}, \mathrm{SnO}_{2}-\mathrm{Sb}_{2} \mathrm{O}_{5}$, and BDD was attributed to the decreasing electrode-hydroxyl radical interaction resulting in a low electrochemical activity for oxygen evolution reaction and to high chemical reactivity for organics oxidation [2]. The effect of anode-hydroxyl radicals interaction is the formation of a layer of radicals that is defined as absorption layer, so the interactions that form this layer requires further attention.

The term adsorption refers to the process by which species are accumulated on the surface of anode and form a layer adjacent to this surface. In the case of an anode, the adsorbed species of the solution must be transported from the bulk of the solution in the adsorption layer, while the species that are produced on the anode are immediately available. When such adsorption is due to the universal weak van der Waals interaction, a multilayer of these species is formed, and it is defined physical adsorption, while when a stronger and more specific interaction occurs between one species and the electrode, it is assumed that a monolayer is adsorbed and the process is defined as chemical adsorption. The interaction which concerns the adsorbed species has been considered by Gibbs [3] by assuming that this layer is two dimensional, that is, a surface. Such treatment, that is characteristic of Gibbs [4] makes easier measurement and studies of energetic aspects of such interfaces. At the same time, this two-dimensional approach is not suitable to study the other aspects that depend on the geometry of this layer.

In the previous paper, it is assumed that the adsorbed species form not a two-dimensional adsorption surface but a three-dimensional thin layer adjacent to the external surface of the anode [5]. In addition, a continuous approach is proposed in this layer that allows to apply the balance equations of the reactive species. Thanks to the use of these tools, the concentrations of reactive species in the adsorption and reactive layer are related to the values of diffusivity and kinetic constants of reactions. The value of the diffusivity 
into the adsorption layer was different in respect to the adjacent reactive layer, while similar values of rate constants were adopted. In this paper, more details on the adsorption and reactive layers will be furnished in order to demonstrate both the possibility to apply the continuum approach in the thin adsorption layer and the validity of the proposed different behaviors of diffusivity and kinetic constant into the adsorption and reactive layers.

\section{Adsorption Layer}

The possibility of the adsorbed species to form a threedimensional adsorption layer and not an adsorption surface has been demonstrated with the use of high-resolution scanning tunnelling microscopy (STM). Thanks to this technique, the adatoms interaction with each other is quantitatively obtained [6]. Such interaction via mutual perturbation of the surface is known as indirect electronic and elastic weak oscillatory interaction $[7,8]$. This is a quantum size effect that induces oscillation in surface charge density near the Fermi level and modulates the adatom surface-binding energies [9]. The effect of the weak and frequent interactions between the adsorbed species have a temporal and spacial smoothing effect on the properties that define this adsorption layer. This smoothing effect is clearly expressed by considering the distribution functions of the various properties that concern this layer. This method is not jet applied in the adsorption field, but such method is not new, and it has been applied in numerous fields [10].

The physical adsorption of species on the various electrodes is due to the universal van der Waals interaction between the electrode and these species. Such interaction between a molecule of this species and the electrode, expressed as potential energy $w_{j}(z)$, is given by [11]:

$$
w_{e, j} z(z)=-\frac{\pi A_{e, j} \rho_{e}}{6} \frac{1}{z^{3}},
$$

where $A_{e, j}$ is a specific constant that relate the considered electrode $e$ with species $j, \rho_{e}$ is the number density of molecules in this anode, and $z$ is the distance between electrode and this molecule. It is interesting to note that the coefficient $A_{e, j}$, of (1), concerns the interaction between the anode $e$ and the species $j$; that is, it expresses the specificity of the action between the electrode and adsorbate.

It is considered the case of an electrolyte that shows the following characteristic $A_{e, j}>A_{e, m}$; that is, the previously reported attractive action is higher for the species $j$ than for the other species $m$. When an electrode is immersed in this electrolyte, the intrinsic chaotic motion of species, due to the temperature, has two opposite effects on the adsorption. The positive effect is that such motion allows the species $j$ to reach the position that optimize the attractive van der Waals interaction. The negative effect is that this motion removes the species $j$ as well as the species $m$ that have just achieved their optimum position. The forward growth rate $r_{f, j}$ of the adsorption concerns the species $j$, and it is related to the attractive effect, while its backward removal rate $r_{b, j}$ is related to the chaotic motion effect. The stationary state of adsorption is reached when the whole growth rate $(r=$ $\left.r_{f, j}-r_{b, j}\right)$ becomes zero, and this occurs when a quantity $n_{a, j}$ of molecules has been adsorbed and that adsorbate species form a thin stationary adsorbed layer of constant thickness $\delta_{a, j}$.

\section{Transition from the Adsorption to the Adjacent Layer}

The main property of the adsorption layer is its higher order in respect to the rest of this solution, and such higher order is attributed to the higher attractive action of the species $j$ of this layer and the electrode $e$. This order property has a high effect on the quality of the transition of this adsorption layer to the adjacent layer. In order to obtain more details on such transition, the attractive potential concerning the thickness $\delta_{j}=z_{1}-z_{0}$ of the adsorbed layer is considered [11]

$$
W_{e, j}\left(\delta_{j}\right)=-\frac{\pi A_{e, j} \rho_{e}}{6} \int_{z_{0}}^{z_{0}+\delta_{j}} \frac{\rho_{j}(z)}{z^{3}} d z,
$$

where $z_{0}$ is the minimum distance electrode-adsorbed species $j$, while $\rho_{j}(z)$ concerns the number of adsorbed molecules of species $j$, and $z$ is the distance from the electrode.

As (2) shows, when the distance $z$ reaches a specific value $z_{0}+\delta_{a, j}$, the van der Waals interaction that locates the molecules in their optimum positions becomes lower than the chaotic motion due to the temperature that removes these species from the achieved optimum positions. The consequence is a reduction of the density $\rho_{j}\left(z_{0}+\delta_{a, j}\right)$, and as (2) points out, it introduces an additional reduction of the van der Waals interaction. The synergism of two negative effects (longer distance and lower density) suggests that when the distance reaches this specific value $\left(\delta_{a, j}\right)$, a rather sharp transition is observed; that is, the layer that is inside this distance is ordered, while outside this layer, the species are subject to a chaotic motion.

Such sharp variation of the properties of adsorption layer assures a good definition of adsorbed layer, and by assuming a constant mean value of density $\rho_{j}(z)$, that is, $\bar{\rho}_{j}=\rho_{j}(z)$, (2) can be integrated, and the attractive potential of the adsorption layer is related to its thickness $\delta_{a, j}$

$$
W_{e, j}\left(\delta_{a, j}\right)=-\frac{\pi A_{e, j} \rho_{e} \bar{\rho}_{j}}{12}\left[\frac{1}{\left(z_{0}+\delta_{a, j}\right)^{2}}-\frac{1}{z_{0}^{2}}\right] .
$$

Experimental results show that the adsorbed $\mathrm{Cu}$ on the $\mathrm{Cu}(111)$ surface can reach a range of over $6 \mathrm{~nm}$, and this interaction decays as a function consistent with the inverse $z^{2}$ dependence [12]. It is interesting to note that also the value of the interaction obtained by (3) shows a similar decay.

\section{Properties of the Adsorption Layer}

As previously reported, the adsorption is represented as a two-dimensional layer, and then, the kinetic theory of particle-on-substrate diffusion is a two-dimensional diffusion; that is, it is assumed that the adsorbate forms a single 
layer of adsorbate, and then the diffusion occurs along such adsorbate surface. Such single layer assumption limits the possibility to represent the diffusion process. The proposed two-dimensional diffusion theory along such single layer represents explicitly the molecule surface interaction as well as accounting for intermolecular interaction. More specifically, the molecule-surface interaction has been represented by assuming not a continuum but a discrete adsorption sites approach characterized by the lattice spacing $l$, the intersite potential barriers $V_{m}$, and the frequency of molecular vibration in the sites $v[13]$.

4.1. Anisotropic Diffusivity into the Adsorption Layer. A preliminary attention is required in order to consider the effect of the morphology of the anode and in particular its external surface on the adsorption. The deviation of such real surface from the geometric plane is commonly defined as roughness. A more exact definition of this surface can be obtained by considering locally its tangent plane, and the deviation of the anodic surface by this tangent plane is defined by the two principal radii of curvature $R_{1}$ and $R_{2}$. Low values of these radii indicate high deviation from the plane and then a high local roughness. If the thickness of the previous defined adsorption layer is lower than curvature radii $\left(\delta_{\mathrm{a}, j} \ll R_{1}\right.$ and $\left.\delta_{a, j} \ll R_{2}\right)$, the normal and parallel directions of the surface of electrode can be defined locally with respective axes $(z, x)$, and the validity of the previous equations (1)-(3) is assured.

If it is assumed that the adsorption layer has a thickness $\delta_{a, j}$, and the diffusivity of the species $j$, in the direction that is parallel to the surface of electrode $D_{a, p, j}(z)$, can be defined, and its value depends on $z$. The mean value of this parallel diffusivity $\bar{D}_{a, p, j}$ is estimated

$$
\bar{D}_{a, p, j}=\frac{\int_{0}^{\delta_{a, j}} D_{a, p, j}(z) d z}{\delta_{a, j}} .
$$

The assumption that the adsorption of species $j$ forms a layer of thickness $\delta_{a, j}$ imposes to consider the diffusion of this species in the direction that is normal to the surface of the electrode. Even in this case, the value of the diffusivity is $D_{a, n, j}(z)$; that is, it depends on the distance $z$, from the anodic surface, and its mean value $\bar{D}_{a, n, j}$ can be estimated as in the case of diffusivity in the direction that is parallel to the electrode of surface.

$$
\bar{D}_{a, n, j}=\frac{\int_{0}^{\delta_{a, j}} D_{a, n, j}(z) d z}{\delta_{a, j}} .
$$

If the mean values of normal diffusivity is different from the parallel diffusivity $\left(\bar{D}_{a, n, j} \neq \bar{D}_{a, p, j}\right)$, the diffusion process of the species $j$ into the adsorption layer is not isotropic.

\subsubsection{Relationship between Surface Diffusivity and Parallel} Diffusivity. The proposed diffusivity in the parallel direction has not yet been considered in this field, because the adsorption layer has been so far represented as a surface $\left(\delta_{a, j} \rightarrow 0\right)$. The diffusivity in this last case is defined as surface diffusivity $D_{s, j}$, and its value must be related to the mean diffusivity in the parallel direction defined in this paper $\bar{D}_{a, p, j}$. In order to obtain this relationship, it is considered the diffusive flow of adsorbate $N_{a, p, j}$ in the adsorption layer according to a direction $x$ that is parallel to the surface of electrode, whose value is

$$
N_{a, p, j}=-\bar{D}_{a, p, j} \frac{d C_{a, V, j}}{d x} \delta_{a, j} L
$$

The flow obtained above is equal to the flow $N_{a, s, j}$ that is estimated considering not a layer but a surface absorption, and its value is

$$
N_{a, s, j}=-D_{a, s, j} \frac{d C_{a, S, j}}{d x} L,
$$

where $C_{a, V, j}$ is the volume concentration used in the case of adsorption layer, $C_{a, S, j}$ is the surface concentration that is used in the case of adsorption surface, and $L$ is the unit length of two- and three-dimensional layers. These concentrations are not independent and their relationship is

$$
C_{a, S, j}=\delta_{a, j} C_{a, V, j} .
$$

By combining (6), (7), and (8), it is obtained that the mean value of the parallel diffusivity introduced in this paper is egual to the value of the surface diffusivity that has been measured experimentally

$$
\bar{D}_{a, p, j}=D_{a, s, j} .
$$

Consequently, the available experimental values as well as the estimated values of the surface diffusivity can be used to estimate the introduced in this paper mean parallel diffusivity.

4.1.2. Comparison between Normal and Parallel Diffusivity. Two different kinds of interactions occurs on the adsorbate $j$, the substrate-adsorbate and the adsorbate-adsorbate interactions due to induced dipole-induced dipole action. The substrate-adsorbate interaction acts principally at a direction that is normal to the surface of the electrode, while the adsorbate-adsorbate interaction acts principally at a direction that is parallel to the anodic surface. The previous consideration on the specificity of these interactions imposes to assume that the substrate-adsorbate interaction is higher in respect to the adsorbate-adsorbate interaction. In other words, the binding action in the direction that is normal to the electrode surface is higher in respect to the binding action in the direction that is parallel to the same surface.

At a higher binding, action corresponds a higher restriction of motion that concern the adsorbed species $j$ and then a lower value of diffusivity [14]. Consequently, the diffusivity of the species $j$ into the adsorption layer is anisotropic and specifically the value of the diffusivity in the normal direction is lower of its value in the parallel direction respect to the surface of electrode

$$
\bar{D}_{a, n, j}<\bar{D}_{a, p, j} .
$$


The diffusion according the parallel diffusion is also known as intralayer or across a step transport, while the diffusion according to the normal direction is known as interlayer transport [15]. Experimental observations with field ion microscope (FIM) confirms the above theoretical conclusion, that is, diffusivity according to the normal direction assumes low values [16]; that is, the diffusivity in the adsorption layer is not a scalar but a vector.

The high contribution of surface diffusion in the whole diffusion process into the porous materials [17] points out that the value of surface diffusivity and then the mean value of diffusivity parallel to electrode surface are relatively high. The anisotropy of the diffusivity in the adsorption layer assures that the concentration value of adsorbate species $j$ is practically constant in the direction that is parallel to the anodic surface $x$, while its value change along the normal direction $\left[C_{a, j}(z)\right]$.

\section{Electrolyte Absent of Hydroxyl Radicals Trappers}

When the anode is operating, the oxidative species $j$ are generated on the anodic surface, and such species enter into the adsorption layer. The escape of these species from the anodic surface is assume instantaneous in this paper. If such species are subjected to attractive action by the anode, its diffusivity $\bar{D}_{a, n, j}$ assumes a low value, and then, the generated species remain for longer time into the adsorption layer. Normally, these oxidative species react between them or oxidise other species, and the consequence of longer permanence in the adsorption layer is the higher consumption of these species. The final effect is that a lower fraction of the generated species escapes from the adsorption layer and achieves the adjacent region. In the case of a BDD anode, the oxidative species are the hydroxyl radicals $\mathrm{HO}^{\bullet}$ that are generated according the following electrochemical reaction $[18,19]$ :

$$
\mathrm{H}_{2} \mathrm{O} \longrightarrow \mathrm{HO}^{\bullet}+\mathrm{H}^{+}+\mathrm{e}^{-}
$$

The flow of the generated hydroxyl radicals is related to the value of the applied anodic current density $i_{\text {appl }}$.

The electrolyte contains other species like biorefractory organic species $R$ in the case of the elimination of this organic species or anions like $\mathrm{SO}_{4}{ }^{2-}$ and $\mathrm{CO}_{3}{ }^{2-}$ in the case of the production of peroxide. The oxidation of these species occurs thanks to the trapping of the freshly generated hydroxyl radicals by these species. In order to point out the principal characteristics of adsorption and adjacent layers, in this paper, the absence of trapped species is assumed.

5.1. Reaction of Hyrdoxyl Radicals into the Adsorption Layer. If the electrolyte is absent of hydroxyl radicals trappers, the generated hydroxyl radicals that enter in the adsorption layer react with each other to produce hydrogen peroxide according to the following reaction:

$$
\mathrm{HO}^{\bullet}+\mathrm{HO}^{\bullet} \longrightarrow \mathrm{H}_{2} \mathrm{O}_{2}
$$

when the rate of the reaction (12) follows the second order law

$$
-r_{a, \mathrm{HO}} \cdot(z)=k_{a, \mathrm{HO}} \cdot C_{a, \mathrm{HO}}^{2} \cdot(z), \quad 0 \leq z \leq \delta_{a, \mathrm{HO}} \cdot
$$

where $k_{a, \mathrm{HO}}$ is the constant of the reaction rate (13) within the adsorption layer and $C_{a, \mathrm{HO}} \cdot(z)$ is the concentration of hydroxyl radicals in the same layer.

5.2. Reaction of Hydroxyl Radicals into the Adjacent Reactive Layer. If the generated radicals are not totally consumed into the adsorption layer by the reaction (12), a portion of the generated radicals escapes from the adsorption layer and achieves the adjacent region when this reaction continues to occur. It is assumed that the rate of the reaction (12) in this layer assume a similar expression:

$$
-r_{a, \mathrm{HO}} \cdot(z)=k_{r, \mathrm{HO}} \cdot C_{a, \mathrm{HO}}^{2} \cdot(z), \quad \delta_{a, \mathrm{HO}} \cdot \leq z \leq \delta_{r, \mathrm{HO}} \cdot
$$

where $k_{r, \mathrm{HO}}$ is the constant of the reaction rate (13) into the reactive layer and $C_{a, \mathrm{HO}}^{2} \cdot(z)$ is the concentration of hydroxyl radicals into this layer.

5.2.1. Rate Constant into the Adsorption and Reactive Layers. It is interesting to note that despite the occurring same reaction (12), different values of reaction rate constant have been assumed for the adsorption and reactive layers $\left(k_{a, \mathrm{HO}} \cdot\right)$ and $\left(k_{r, \mathrm{HO}}\right)$. The different values have been assumed, because the reaction into the adsorption layer concerns hydroxyl radicals that are partially embedded by the attractive action electrode-hydroxyl radicals, while the same reaction into the reactive layer concerns hydroxyl radicals that are freely moved into the electrolyte solution.

Other conditions being equal, an increase in diffusivity follows an increase in the chaotic movement of species that react with an increase in collisions between them, and thus the increase of the reaction rate constant. As previously reported, the diffusivity in the adsorption layer is anisotropic, and then, the principal contribution on the reaction rate is due to the high value of chaotic motion that is parallel to the surface of electrode. In the adjacent reactive layer, the diffusivity is isotropic, and its value is surely higher than the normal diffusivity in the adsorption lower and probably higher than the parallel diffusivity, and then, the comparison of the collisions in the two layers is not an easy task.

\section{Adsorption, Reaction, and Diffusion Layers}

In the adsorption layer, two events characterize this layer, the interactions in hydroxyl radicals electrode and the reaction (12), while the adjacent layer is characterized by the occurring of the reaction (12), and for this reason, it is defined as reactive layer. The events that characterize both adsorption and reaction layer concern the generated hydroxyl radicals on the surface of the anode. Consequently, the existence of the two layers depends on the rate of the generated radicals, and when the anodic surface does not generate hydroxyl radicals, both radicals are absent

$$
\delta_{a, \mathrm{HO}}=\delta_{r, \mathrm{HO}}=0 .
$$




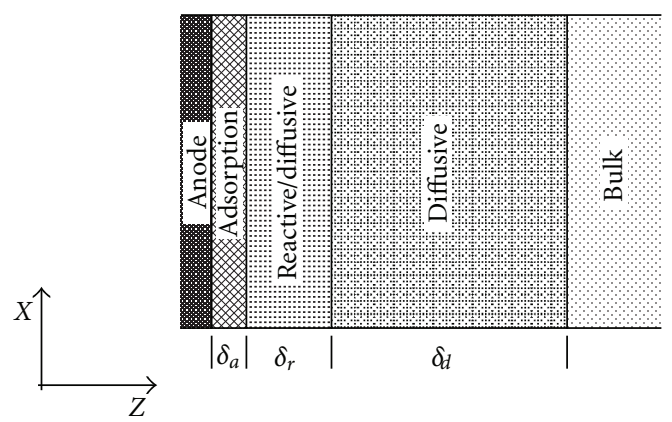

FIGURE 1: Scheme of anode with the adjacent layers of the solution (not in scale).

While when this generation rate is high, both adsorption and reaction layers are present

$$
0 \leq \delta_{a, \mathrm{HO}^{\bullet}} \leq \delta_{r, \mathrm{HO}^{\circ}} .
$$

In fact, in the last case, a high number of hydroxyl radicals are generated, and a fraction of these radicals are not transformed to hydrogen peroxide in the adsorption layer according to the reaction (12), and such fraction achieves the adjacent reactive layer, where it reacts and form hydroxyl peroxide.

The two adjacent layers adsorption and reaction are schematically indicated in Figure 1, along with the diffusion layer of thickness $\delta_{d}$. In this case, through such layer, the generated hydrogen peroxide is transported to the bulk of the electrolyte.

The adopted continuum approach on the adsorption and reactive layers allows to apply the differential balance of hydroxyl radical, and the equation of this balance assumes the following form in the adsorption layer [18]:

$$
\bar{D}_{a, n, \mathrm{HO}} \cdot \frac{d^{2} C_{a, \mathrm{HO}}}{d z^{2}}=k_{a, \mathrm{HO}} \cdot C_{a, \mathrm{HO}}^{2} ; \quad 0 \leq z \leq \delta_{a},
$$

while a similar equation is obtained for the subsequent reactive layer

$$
D_{r, \mathrm{HO}} \cdot \frac{d^{2} C_{r, \mathrm{HO}}}{d z^{2}}=k_{r, \mathrm{HO}} \cdot C_{r, \mathrm{HO}}^{2} ; \quad \delta_{a} \leq z \leq \delta_{r} .
$$

The balance equation that concerns the adsorption layer assumes the following dimensionless form:

$$
\frac{d^{2} y_{a}}{d \xi_{a}^{2}}=y_{a}^{2} ; \quad 0 \leq \xi_{a} \leq \frac{\delta_{a}}{\lambda_{a}},
$$

while a formally similar equation is obtained for the successive reactive layer

$$
\frac{d^{2} y_{r}}{d \xi_{r}^{2}}=y_{r}^{2} ; \quad \frac{\delta_{a}}{\lambda_{a}} \leq \xi_{r} \leq \frac{\delta_{r}}{\lambda_{a}},
$$

where $y_{a}$ and $y_{r}$ are the dimensionless concentrations of hydroxyl radicals into the adsorption and reactive layers

$$
y_{a}=\frac{C_{\mathrm{HO}^{*}, a}}{C_{\mathrm{HO}} \cdot(0)}, \quad y_{r}=\frac{C_{\mathrm{HO}^{*}, r}}{C_{\mathrm{HO}} \cdot(0)},
$$

where $C_{\mathrm{HO}}(0)$ is the concentration of hydroxyl radical in the region of the adsorption layer that is adjacent to the electrode. Two characteristic parameters were introduced having the dimension of length, the first concerning the adsorption layer $\lambda_{a}$, and the second concerning the reactive layer $\lambda_{r}$. Such parameters are obtained by combining the various parameters of (17) and (18)

$$
\lambda_{a}=\sqrt{\frac{\bar{D}_{a, n, \mathrm{HO}}}{k_{a, \mathrm{HO}} \cdot C_{\mathrm{HO}}(0)}} ; \quad \lambda_{r}=\sqrt{\frac{D_{r, \mathrm{HO}}}{k_{\mathrm{HO}} \cdot C_{\mathrm{HO}}(0)}} .
$$

The above parameters are defined as characteristic lengths of the adsorption $\lambda_{a}$, and reactive layer $\lambda_{r}$ and these lengths are used to define the two dimensionless distances: $\xi_{a}=$ $z / \lambda_{a}$ and $\xi_{r}=z / \lambda_{r}$. The characteristic length of reactive layer $\lambda_{r}$ is related to parameters that are usually available, and by considering a diffusion coefficient $D_{r, \mathrm{HO}}{ }^{*}=2.2$. $10^{-9} \mathrm{~m}^{2} \mathrm{~s}^{-1}$, a rate constant $k_{\mathrm{HO}} \cdot=5.5 \cdot 10^{6} \mathrm{~mol}^{-1} \mathrm{~s}^{-1}$, and a concentration $C_{\mathrm{HO}} \cdot(0)=0.1 \mathrm{~mol} \mathrm{dm}^{-3}$, the value of the characteristic length of reactive layer is $32 \mathrm{~nm}$ [5], while the estimation of characteristic length $\lambda_{a}$ that concerns the adsorption layer is more complicate. In this paper, it is assumed that all the generated hydroxyl radicals on the anodic surface achieve immediately the adjacent region of adsorption layer. The value of $\lambda_{a}$ is a measure of survival distance of hydroxyl radicals while they moved into the adsorption layer, while the value of $\lambda_{r}$ is a measure of survival distance in the reactive layer.

6.1. Adsorption Layer and Adsorption Parameter. The thickness of the adsorption layer $\delta_{a}$ is a parameter that define the geometry of this layer, but this value is not enough to relate adsorption state of hydroxyl radicals with the reaction and diffusion. An ulterior parameter is required that is obtained by considering the ratio of the previously introduced characteristic adsorption and reactive layers

$$
\beta=\frac{\lambda_{a}}{\lambda_{r}}=\sqrt{\frac{\bar{D}_{a, n, \mathrm{HO}} \cdot}{D_{r, \mathrm{HO}} \cdot} \frac{k_{r, \mathrm{HO}}}{k_{a, \mathrm{HO}}} .} .
$$

This ratio is also a dimensionless number that compares the known constants of the two mechanisms in the reactive layer to the responding unknown constants into the adsorption layer.

In this paper, it is assumed that the values of rate constants in the two layers are approximately equal $\left(k_{a, \mathrm{HO}} \approx\right.$ $\left.k_{r, \mathrm{HO}}\right)$, while the value of the normal diffusivity in the adsorption layer is smaller than the diffusivity value in the reactive layer $\left(\bar{D}_{a, n, \mathrm{HO}}<D_{r, \mathrm{HO}}\right)$. The values of $\beta$ is, therefore, between zero and one $(0<\beta<1)$, and when $\beta \rightarrow 1$, the effect of the adsorption is very weak, while this effect becomes strong when $\beta \rightarrow 0$.

The analytic solution of (19) has been obtained in the previous paper [5], and such solution in the adsorption layer assumes the following form:

$$
y_{a}=\frac{1}{\left(\sqrt{8 / 3} \xi_{a}+1\right)^{2}} ; \quad 0 \leq \xi_{a} \leq \frac{\delta_{a}}{\lambda_{a}} .
$$




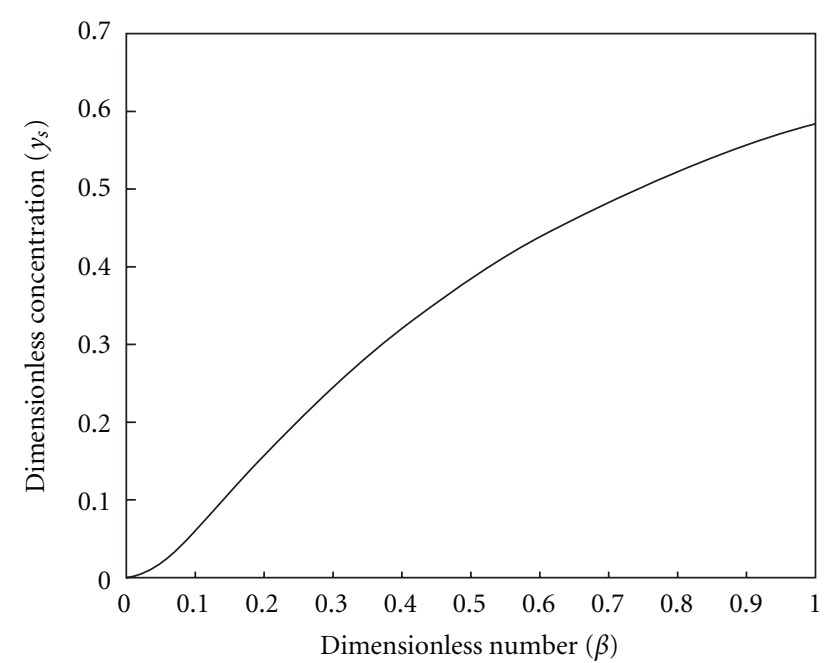

FIgURE 2: Dimensionless concentration $y_{a}$ versus dimensionless number $\beta\left(\delta=6 \mathrm{~nm}\right.$ and $\left.\lambda_{r}=32 \mathrm{~nm}\right)$.

Equation (24), that represents the dimensionless concentration of hydroxyl radical, is calculated at the outside of the adsorption layer when $\delta_{a}=6 \mathrm{~nm}$ and $\lambda_{r}=32 \mathrm{~nm}$ and various values of $\beta$.

Figure 2 reports the obtained concentration outside the adsorption layer $\left(\xi_{a}=\delta_{a} / \lambda_{a}\right)$. These results indicates that when the adsorbing property of this layer becomes strong $(\beta \rightarrow 0)$, all the generated hydrogen radicals on the anodic surface are eliminated on the adsorption layer, and the hydroxyl radical cannot be detected. In the opposite condition, that is, when the value of $\beta \rightarrow 1$, as in the case of BDD anodes, the adsorbing property is very weak, a high portion of the generated radicals escape from the adsorption layer, and these radicals can be detected by using suitable trappers of these radicals, as N,N-dimethyl-p-nitrosoaniline [20]. It is evident that also the organic compounds as well as anions like sulfate and carbonate trap the hydroxyl radicals.

\section{Conclusions}

The high oxidative action of BBD anodes has been attributed to its property to adsorb loosely the generated hydroxyl radicals on its surface. This paper contains more elements of the continuum approach theory of hydroxyl radicals adsorption proposed in a previous paper. Such continuum approach allows to obtain many details on the occurring mechanisms into the adsorption layer unobtainable by other approaches. The critical point of such continuum approach is its apparent contradiction with the continuity condition of the properties of this thin layer.

Recent scanning tunnelling microscopy results allow to determine the thickness of this adsorption layer as well as its dynamic state that has quantum size properties (weak oscillating interactions). For an absorbing film, with a such dynamic state, the perturbation theory suggests to represent its particles and its properties in terms of distribution functions. These distribution functions are continuous, and then, the previously reported contradiction has been avoided thanks to the use of such functions.

In the previous paper, it is assumed that the diffusivity in the adsorption layer is lower than in the adjacent reactive layer. The attractive interaction electrode-hydroxyl radical and mobility of these radicals has been considered in this paper, and such interaction between attraction and mobility points out the validity of the previous assumption on the diffusivity. Moreover, this interaction suggests that the diffusivity in the adsorption layer has a anisotropic property, and more precisely, it is a vector.

In this paper, the adsorption layer has been characterized by its thickness and parameters of diffusivity and rate constants in respect to the adjacent reactive layer.

The fraction of generated hydroxyl radicals that escapes from the adsorption layer and achieves the adjacent reactive layer has been estimated at different values of adsorbing parameter. The obtained results points clearly out the capacity of BBD to generate hydroxyl radicals that are not consumed in the adsorption layer, and then, they are available to oxidize other organic or inorganic species.

\section{References}

[1] J. Iniesta, P. A. Michaud, M. Panizza, G. Cerisola, A. Aldaz, and C. Comninellis, "Electrochemical oxidation of phenol at boron-doped diamond electrode," Electrochimica Acta, vol. 46, no. 23, pp. 3573-3578, 2001.

[2] A. Kapalka, G. Fóti, and C. Comninellis, "Basic principles of the electrochemical mineralization of organic pollutants for wastewater treatment," in Electrochemistry for the Environment, C. Comninellis and G. Chen, Eds., Springer, New York, NY, USA, 2010.

[3] J. W. Gibbs, Collected Works, vol. 1, Longmans, London, UK, 1928.

[4] E. Schrödinger, Statistical Thermodynamics, CUP, London, UK, 1946.

[5] N. Vatistas, "Adsorption layer and its characteristic to modulate the electro-oxidation runway of organic species," Journal of Applied Electrochemistry, vol. 40, no. 10, pp. 1743-1750, 2010.

[6] L. Österlund, M. Ø. Pedersen, I. Stensgaard, E. Lægsgaard, and F. Besenbacher, "Quantitative determination of adsorbateadsorbate interactions," Physical Review Letters, vol. 83, no. 23, pp. 4812-4815, 1999.

[7] T. T. Tsong, C. S. Chang, I. S. Hwang et al., "Electron and atom dynamics at solid surfaces and relation to epitaxy," Journal of Physics and Chemistry of Solids, vol. 62, no. 9-10, pp. 16891730, 2001.

[8] N. Knorr, H. Brune, M. Epple, A. Hirstein, M. A. Schneider, and K. Kern, "Long-range adsorbate interactions mediated by a two-dimensional electron gas," Physical Review B, vol. 65, no. 11, article 115420, 2002.

[9] L.-Y. Ma, L. Tang, Z.-L. Guan et al., "Quantum size effect on adatom surface diffusion," Physical Review Letters, vol. 97, no. 26, article 266102, 2006.

[10] J. Awrejcewicz, I. V. Andrianov, and L. I. Manevitch, Asymptotic Approaches in Nonlinear Dynamics, Springer, Berlin, Germany, 1998.

[11] J. N. Israelachvili, Intermolecular and Surface Forces, Academic Press, London, UK, 1992. 
[12] J. Repp, F. Moresco, G. Meyer, and K. H. Rieder, "Substrate mediated long-range oscillatory interaction between adatoms: $\mathrm{Cu} / \mathrm{Cu}(111)$," Physical Review Letters, vol. 85, no. 14, pp. 29812984, 2000.

[13] S. Y. Krylov, J. J. M. Beenakker, and M. C. Tringides, "On the theory of surface diffusion: kinetic versus lattice gas approach," Surface Science, vol. 420, no. 2-3, pp. 233-249, 1999.

[14] S. Miret-Artés and E. Pollak, "The dynamics of activated surface diffusion," Journal of Physics-Condensed Matter, vol. 17, no. 49, p. S4133, 2005.

[15] H. Ibach, Physics of Surfaces and Interfaces, Springer, Berlin, Germany, 2006.

[16] G. Ehrlich and F. G. Hudda, "Atomic view of surface selfdiffusion: tungsten on tungsten," The Journal of Chemical Physics, vol. 44, no. 3, pp. 1039-1049, 1966.

[17] K. Miyabe and G. Guiochon, "Measurement of the parameters of the mass transfer kinetics in high performance liquid chromatography," Journal of Separation Science, vol. 26, no. 34, pp. 155-173, 2003.

[18] A. Kapałka, G. Fóti, and C. Comninellis, "The importance of electrode material in environmental electrochemistry: formation and reactivity of free hydroxyl radicals on borondoped diamond electrodes," Electrochimica Acta, vol. 54, no. 7, pp. 2018-2023, 2009.

[19] T. A. Enache, A. M. Chiorcea-Paquim, O. Fatibello-Filho, and A. M. Oliveira-Brett, "Hydroxyl radicals electrochemically generated in situ on a boron-doped diamond electrode," Electrochemistry Communications, vol. 11, no. 7, pp. 13421345, 2009.

[20] C. Comninellis, "Electrocatalysis in the electrochemical conversion/combustion of organic pollutants for waste water treatment," Electrochimica Acta, vol. 39, no. 11-12, pp. 18571862, 1994. 


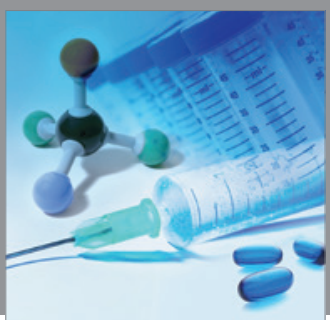

International Journal of

Medicinal Chemistry

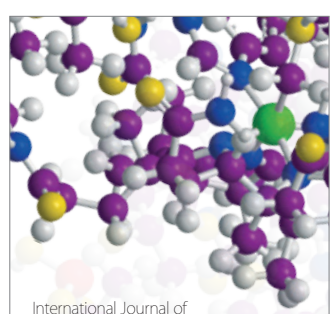

Carbohydrate Chemistry

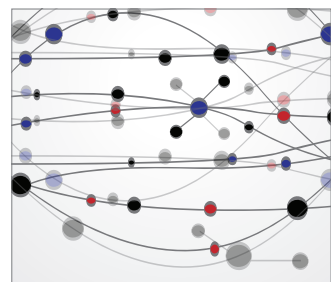

The Scientific World Journal
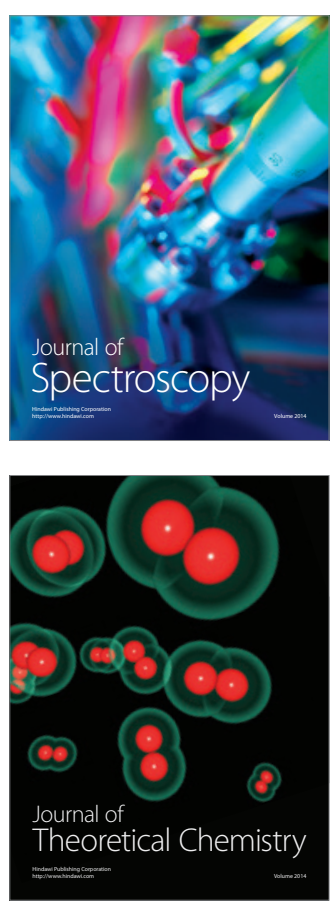
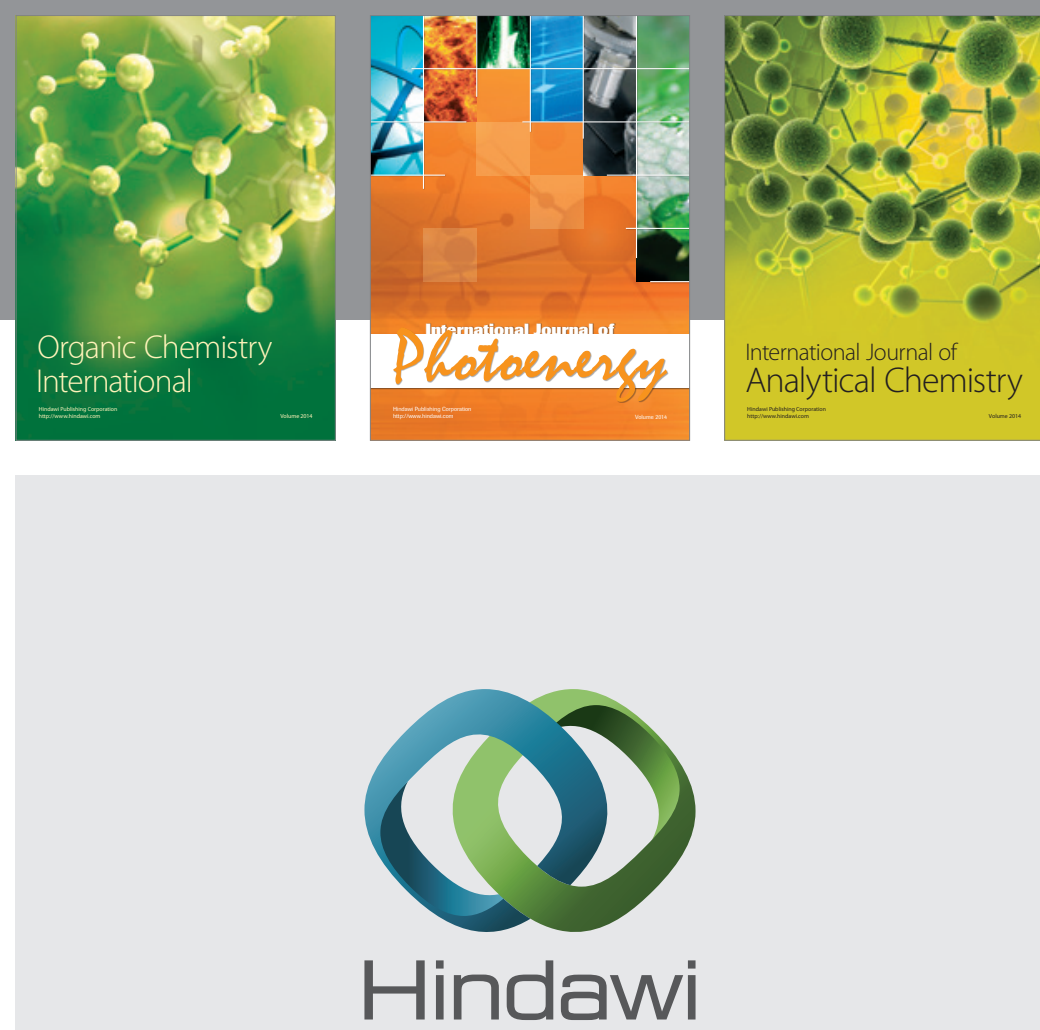

Submit your manuscripts at

http://www.hindawi.com
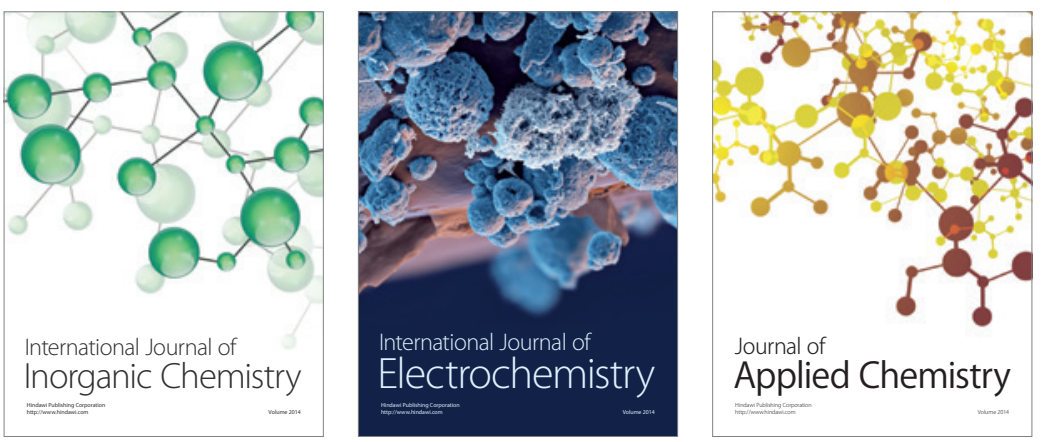

Journal of

Applied Chemistry
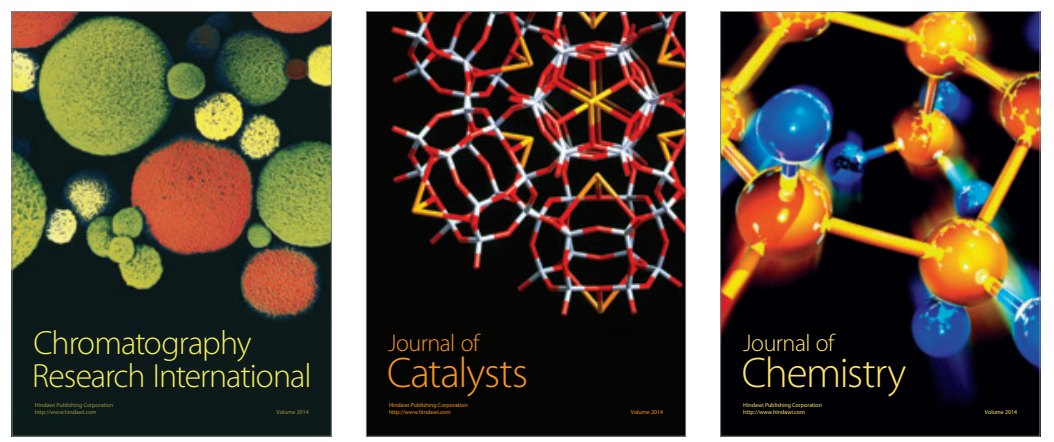
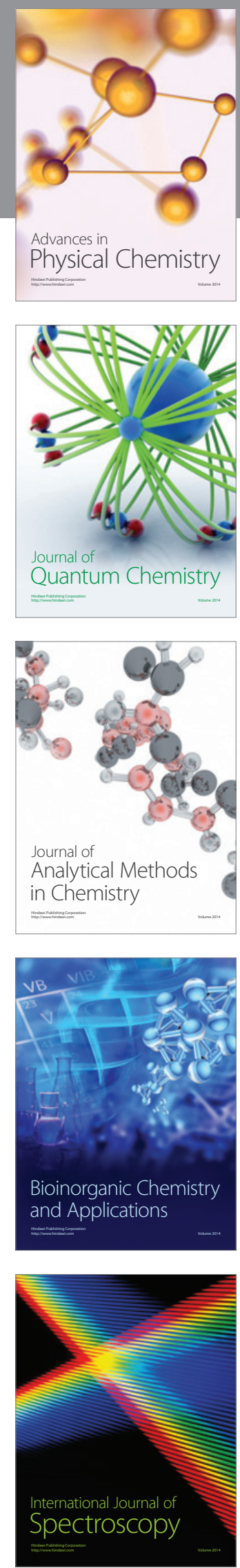